\title{
DISEÑO DE UN PROTOTIPO DE SISTEMA ANTI-VUELCO PARA VEHÍCULOS INDUSTRIALES
}

\author{
Jesús Molina, Mario L. Ruz, Francisco Vázquez \\ Dpto. de Informática y Análisis Numérico, Universidad de Córdoba, Campus de Rabanales, Edificio \\ Leonardo Da Vinci, Córdoba, España \\ fvazquez@uco.es
}

\begin{abstract}
Resumen
En este trabajo se ha desarrollado un prototipo de sistema anti-vuelco para vehículos industriales. En primer lugar, se realizó un estudio sobre el estado del arte de este tipo de dispositivos, recopilándose información sobre indices que estiman la estabilidad de un vehículo. A partir de dicho estudio, se seleccionó el hardware necesario para la implementación de algoritmos que calculan estos indices en tiempo real. El prototipo diseñado dispone de una alarma sonora, una salida a relé, capacidad de registro de datos y una interfaz gráfica. Además, es posible transmitir los indices de estabilidad calculados mediante bus CAN, lo que permite una integración del equipo con los sensores $y$ mecanismos de control del vehículo donde se instale. Para la evaluación del prototipo se llevaron a cabo un conjunto de pruebas tanto de simulación como reales. Los resultados obtenidos ponen de manifiesto la importancia del desarrollo e investigación de este tipo de equipos en la reducción de accidentes por vuelco.
\end{abstract}

Palabras Clave: Sistema anti-vuelco, Estabilidad, Vehículo agrícola, bus CAN.

\section{INTRODUCCIÓN}

La existencia de accidentes graves y mortales por vuelco de tractores y maquinaria agrícola es un problema que preocupa a nivel europeo. Las causas de estos accidentes son varias, como maniobras incorrectas en el manejo del vehículo, o la peligrosidad intrínseca de las condiciones de trabajo (falta de estabilidad del vehículo, configuración irregular del terreno, etc.).

Dentro del sector agrario, gran parte de los accidentes mortales se producen por pérdida del control de maquinaria y vehículos agrícolas. En España, el 55 \% de los accidentes mortales producidos en este sector se debe a la casuística comentada [1]. Es por ello que se pretende buscar una solución a la problemática actual, con el objetivo principal de mejorar la seguridad de los trabajadores en el uso de vehículos con alto riesgo de siniestralidad por vuelco.

En este aspecto, es fundamental el desarrollo de nuevos dispositivos anti-vuelco que permitan una mejora sustancial en la prevención de los accidentes comentados. Se considera por tanto de interés el desarrollo de un prototipo que disminuya el número de accidentes por vuelco y que aporte características adicionales a equipos ya disponibles en el mercado. En este trabajo se ha desarrollado un prototipo que pretende, por un lado, servir como plataforma de pruebas de diferentes algoritmos anti-vuelco, y por otro, integrar toda la información que se calcule con el sistema de control del propio vehículo mediante bus CAN (Controller Area Network).

El resto del artículo se organiza de la siguiente manera: en la sección 2 se explican los índices de estabilidad recopilados de la bibliografía consultada e implementados en el prototipo elaborado. En la sección 3 se describe dicho prototipo y se comentan los principales componentes de los que consta. En la sección 4 se presentan las distintas pruebas realizadas para su evaluación, destacándose una prueba real en un vehículo agrícola. Finalmente, en la sección 5 se resumen las conclusiones y las líneas de continuación de este trabajo.

\section{2 ÍNDICES DE ESTABILIDAD}

Para determinar una situación de peligro relacionada con un posible vuelco es necesario recurrir a algoritmos que indiquen de alguna forma la estabilidad del vehículo, y de este modo actuar en consecuencia. Existen distintos algoritmos en la bibliografía consultada [2], [3], [4] [5], que a partir de la orientación del vehículo y distintos parámetros mecánicos del mismo proporcionan una medida de la estabilidad frente a un posible vuelco.

Los índices presentados en este trabajo dan como resultado un valor entre 0 y 100 , siendo 100 el correspondiente a la máxima estabilidad, es decir, la situación en la cual el vehículo se encuentra en un régimen estático (velocidades angulares nulas) en un plano sin pendiente. El valor 0 representa el límite a 
partir del cual se considera que el vehículo se encuentra en una situación de inestabilidad.

Los índices de estabilidad se pueden clasificar en dos grupos: estáticos y dinámicos. Los primeros proporcionan una medida de la estabilidad en función de la orientación del vehículo y parámetros mecánicos del mismo (peso, dimensiones del vehículo, altura del centro de gravedad, etc). La inclinación del vehículo (véase la Figura 1) se puede definir a partir de tres ángulos (pitch, roll, yaw) respecto a un sistema de referencia ortonormal con origen en el centro de gravedad del vehículo (O). El pitch o cabeceo representa una inclinación a lo largo del eje longitudinal del tractor, el roll o alabeo representa una rotación a lo largo del mismo eje, y el ángulo de guiñada o yaw una rotación respecto a un eje perpendicular al vehículo.

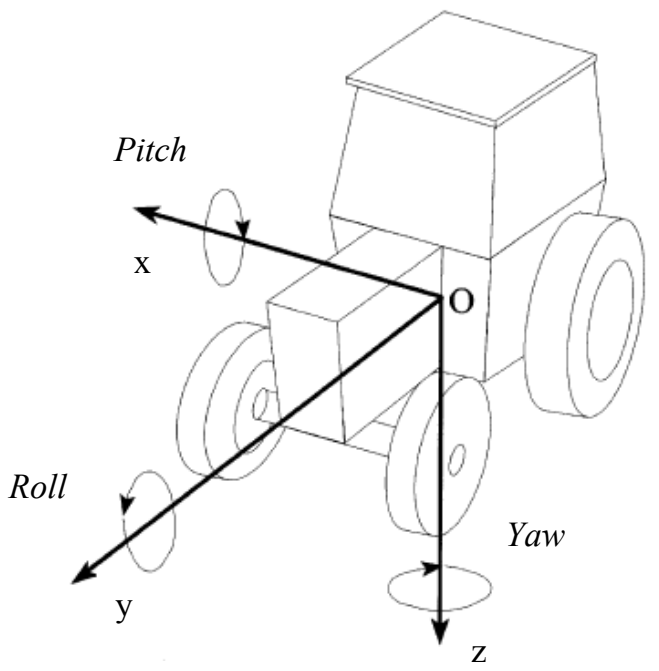

Figura 1: Ángulos de orientación

Para el cálculo de los índices dinámicos además de lo mencionado anteriormente son necesarios las velocidades angulares del tractor y los momentos de inercia con respecto a sus ejes.

\section{1 ÍNDICES DE ESTABILIDAD ESTÁTICOS}

Se han recopilado varios índices de estabilidad estáticos en función del tipo de vuelco. Se distinguen tres: el índice de estabilidad longitudinal ( $\left.\mathrm{SI}_{\mathrm{LONG}}\right)$, el índice de estabilidad lateral ( $\mathrm{SI}_{\mathrm{LATS}}$ ) y el índice de estabilidad de giro lateral ( $\left.\mathrm{SI}_{\text {LATT }}\right)$. En todos los índices mostrados en este trabajo se utiliza el Sistema Internacional de Unidades.

El primero de ellos, $\left(\mathrm{SI}_{\mathrm{LONG}}\right)$, determina la estabilidad del vehículo en función de su inclinación a lo largo del eje principal del tractor (eje longitudinal), y viene determinado por la siguiente ecuación:

$$
S I_{L O N G}=\left(1-\frac{\theta}{\theta_{c r i}}\right) \cdot 100,
$$

donde $\theta(\mathrm{rad})$ representa el ángulo de inclinación del eje longitudinal, y $\theta_{c r i}(\mathrm{rad})$ el ángulo de inclinación crítico, a partir de la cual el tractor es inestable, definida por la siguiente ecuación:

$$
\theta_{c r i}=\tan ^{-1}\left(\frac{w_{b}}{2 h}\right),
$$

donde $w_{b}(\mathrm{~m})$ representa la distancia entre los ejes de las ruedas delanteras y traseras del vehículo, y $h(\mathrm{~m})$ la altura del centro de gravedad.

Por otra parte, en el índice $\mathrm{SI}_{\mathrm{LATS}}$ se considera la inclinación lateral del tractor. Viene dado por la ecuación (3):

$$
S I_{\text {LATS }}=\left(1-\frac{\phi}{\phi_{c r i}}\right) \cdot 100 \text {, }
$$

Donde $\phi(\mathrm{rad})$ representa el ángulo de inclinación lateral y $\phi_{c r i}(\mathrm{rad})$ el ángulo de inclinación lateral crítico, definido en la ecuación (4), a partir de la cual el tractor se considera inestable:

$$
\theta_{c r i}=\tan ^{-1}\left(\frac{2 h}{t_{w}}\right),
$$

donde $t_{w}(\mathrm{~m})$ representa el ancho de vía del tractor y $h(\mathrm{~m})$ la altura de su centro de gravedad.

El tercero de los índices estáticos contemplados es el

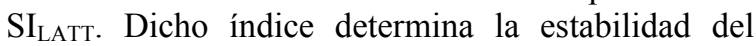
vehículo cuando éste realiza un giro en una pendiente. $\mathrm{SI}_{\text {LATT }}$ viene definido por la siguiente ecuación:

$$
S I_{\text {LATT }}=\left(1-\frac{V}{V_{c r i}}\right) \cdot 100,
$$

donde $V\left(\mathrm{~m} \cdot \mathrm{s}^{-1}\right)$ representa la velocidad del tractor y $V_{c r i}\left(\mathrm{~m} \cdot \mathrm{s}^{-1}\right)$ la velocidad crítica, a partir de la cual se considera que el tractor deja de ser estable. $V_{c r i}$ viene definida por la siguiente ecuación:

$$
V_{c r i}=\sqrt{\frac{g \cdot R \cdot A}{Z_{c g} \cdot \cos \gamma}},
$$

donde $g$ representa la aceleración de la gravedad $\left(\mathrm{m} \cdot \mathrm{s}^{-2}\right), R(\mathrm{~m})$ el radio de giro, $A(\mathrm{~m})$ es la distancia horizontal más corta en el plano entre el centro de 
gravedad y un plano vertical que pasa por el denominado tipping axis, que se define como el eje a partir del cual el vehículo volcaría lateralmente. Por último, $Z_{c g}(\mathrm{~m})$ representa la altura del centro de gravedad y $\gamma$ es el ángulo formado entre el tipping axis y el plano longitudinal [5].

\subsection{1 Índice de estabilidad combinado}

Durante el uso de un vehículo agrícola pueden existir situaciones en las que se comprometa su estabilidad tanto longitudinal como lateralmente, y además de forma simultánea. El índice de estabilidad combinado SI $I_{\text {COM }}$ considera tanto $\mathrm{SI}_{\text {LONG }}$ como $\mathrm{SI}_{\text {LATT }}$ de forma conjunta. Viene dado por la siguiente ecuación:

$$
S I_{C O M}=\left(1-\sqrt{\frac{\phi^{2}}{\phi_{c r i}^{2}}+\frac{\theta^{2}}{\theta_{c r i}^{2}}}\right) \cdot 100
$$

Como se puede observar, este índice de estabilidad utiliza los parámetros empleados en las ecuaciones (1) y (3), combinando los ángulos de pitch y roll así como sus valores críticos. Se puede observar fácilmente que en el momento en el cual uno de los ángulos sea igual a su valor crítico el índice $\mathrm{SI}_{\mathrm{COM}}$ tomaría como máximo el valor de cero, indicándose por tanto una situación de inestabilidad.

\section{2 ÍNDICE DE ESTABILIDAD DINÁMICO}

Los índices anteriores no son suficientes para determinar la estabilidad del tractor, ya que en situaciones reales se pueden producir variaciones bruscas en la inclinación debido a la irregularidad del terreno. Estas variaciones evidentemente son situaciones no estáticas que afectan a la estabilidad del tractor. Por tanto, también es necesario evaluar otros parámetros, como los momentos de inercia con respecto a los ejes y las velocidades angulares producidas, dando lugar a los índices de estabilidad de tipo dinámico.

El índice de estabilidad dinámico, $\mathrm{SI}_{\mathrm{DIN}}$, determina la estabilidad del vehículo en función de las velocidades angulares, momentos de inercia con respecto a los ejes, el ángulo de inclinación lateral y el ángulo de inclinación longitudinal. Se define en la siguiente ecuación:

$$
S I_{D I N}=\left(1-\sqrt{\frac{\phi^{2}}{\phi_{c r i}^{2}}+\frac{\theta^{2}}{\theta_{c r i}^{2}}}\right) \cdot\left(1-\sqrt{\frac{\dot{\phi}^{4}}{\dot{\phi}_{c r i}^{4}}+\frac{\dot{\theta}^{4}}{\dot{\theta}_{c r i}^{4}}}\right) \cdot 100
$$

Como se puede observar, este índice también utiliza parámetros utilizados en los anteriores índices, además de las velocidades angulares

correspondientes, $\phi_{c r i}$ y $\theta_{c r i}$. De esta forma, la medida de estabilidad no solo se ve afectada por los ángulos de orientación, sino también por la velocidad a la que éstos varían. Las velocidades angulares críticas se definen en las siguientes ecuaciones:

$$
\begin{aligned}
& \dot{\phi}_{c r i}=\left(\sqrt{\frac{2 \cdot m \cdot g \cdot r_{x} \cdot\left(1-\cos \left(\phi_{c r i}-\phi\right)\right)}{I_{x x}+m \cdot r_{x}^{2}}}\right) \\
& \dot{\theta}_{c r i}=\left(\sqrt{\frac{2 \cdot m \cdot g \cdot r_{y} \cdot\left(1-\cos \left(\theta_{c r i}-\theta\right)\right)}{I_{y y}+m \cdot r_{y}^{2}}}\right),
\end{aligned}
$$

donde $m(\mathrm{~kg})$ representa la masa del tractor, $g$ la aceleración de la gravedad $\left(\mathrm{m} \cdot \mathrm{s}^{-2}\right), r_{x}$ y $r_{y}$ vienen definidas por las ecuaciones (11) y (12), respectivamente, y cuyos parámetros ya se han definido anteriormente. Por otra parte, $I_{x x}$ e $I_{y y}$ representan los momentos de inercia respecto a los ejes $x$ e $y$, respectivamente (véase la Figura 1).

$$
\begin{aligned}
& r_{x}=\left(\frac{t_{w}}{2}\right)^{2}+h^{2} \\
& r_{y}=\left(\frac{w_{b}}{2}\right)^{2}+h^{2}
\end{aligned}
$$

\section{DESARROLLO DEL PROTOTIPO}

Como ya se comentó, el objetivo principal de este trabajo es el desarrollo de un prototipo de sistema anti-vuelco para vehículos industriales o agrícolas. Dicho dispositivo debe calcular en tiempo real los índices de estabilidad descritos en la sección anterior, los cuales se basan esencialmente en medidas de ángulos que determinan la orientación del vehículo, velocidades de los mismos, y parámetros característicos de éste. En la Figura 2 se muestra un esquema general del dispositivo desarrollado.

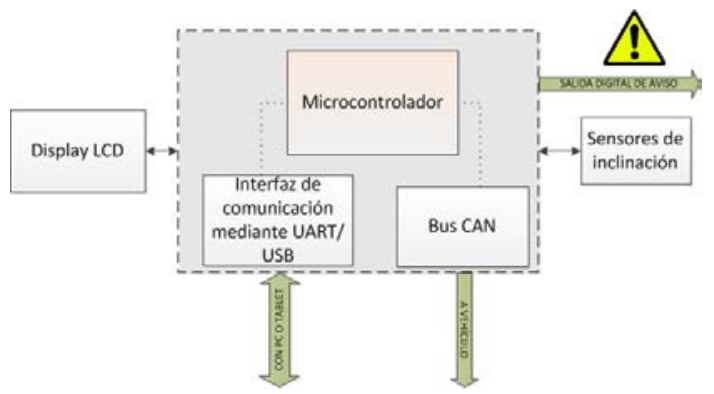

Figura 2: Esquema general del prototipo 
El sistema desarrollado tiene como principal funcionalidad informar sobre la posibilidad de riesgo de vuelco, realizando este aviso mediante una alarma sonora cuando la condición de inestabilidad se cumple. Esta alarma también sirve para notificar al conductor de una situación próxima a la inestabilidad, emitiendo un sonido intermitente cuya frecuencia aumenta al disminuir el índice de estabilidad seleccionado. El dispositivo también cuenta con una salida a relé, la cual se ha incorporado para aquellos casos en los que se quiera activar un segundo sistema de emergencia que realice una acción en el vehículo o active algún tipo de alarma adicional, como puede ser otra señal de aviso. El relé se activará cuando se haya superado un límite inferior de estabilidad definido por programa.

Por otra parte, se ha incorporado un lector de tarjetas tipo SD para almacenar en tiempo real los datos de los índices de estabilidad. Esta característica permite un análisis offline para el estudio de la estabilidad del vehículo y también para comprobar el correcto funcionamiento del dispositivo.

Con la incorporación de un display el usuario puede monitorizar los índices de estabilidad. Se han programado diferentes pantallas, donde se muestran los ángulos de orientación actuales del vehículo, unas barras indicativas de la estabilidad según varios índices calculados (seleccionables por el usuario), y la posibilidad de modificar los parámetros que se utilizan en el cálculo de dichos índices. De esta manera es posible configurar el equipo en función del vehículo donde se instale. El prototipo además cuenta con una interfaz para la comunicación por bus CAN. Se decidió implementar este tipo de interfaz debido al extendido uso de este protocolo en aplicaciones automóviles.

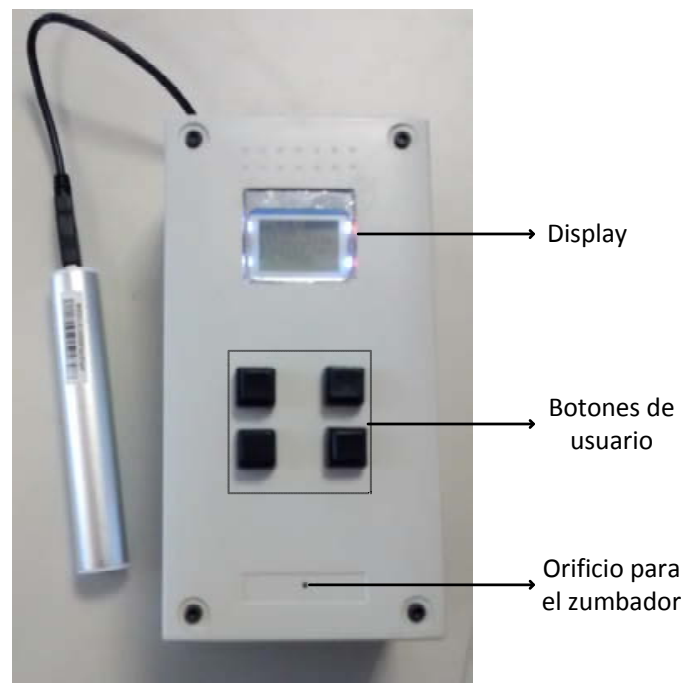

Figura 3: Cara superior del dispositivo
En la Figura 3 se muestra una imagen del dispositivo construido, donde se observa además del display para la visualización, los botones de usuario para el manejo de la interfaz gráfica, un orificio para que el sonido de la alarma sonora sea audible desde el exterior y un cable USB que será utilizado para la alimentación del dispositivo. En las zonas laterales se ubican las conexiones para la tarjeta $\mathrm{SD}$, la salida a relé, y la comunicación CAN.

\section{PRUEBAS REALIZADAS}

El dispositivo desarrollado ha sido sometido a un conjunto de pruebas: simuladas, basadas en datos registrados en la tarjeta $\mathrm{SD}$; y en situaciones reales, ejecutando los algoritmos en el prototipo. En este último caso se ha empleado un periodo de muestreo de las medidas de los sensores (ángulos de orientación y sus velocidades) de $0.1 \mathrm{~s}$. Cada vez que se actualizan dichas medidas se vuelven a calcular los índices de estabilidad.

\subsection{COMUNICACIÓN CAN}

Una de las pruebas realizadas consistió en el estudio de la funcionalidad de la interfaz CAN. Para ello, se utilizó un segundo microcontrolador de similares características al utilizado para el desarrollo del prototipo. Este segundo microcontrolador simula un vehículo que dispone de conectividad por bus CAN.

La prueba consistió en la transmisión del valor de la velocidad por parte del microcontrolador que simula el vehículo. Este valor es recibido por el prototipo y a partir de este dato, de los ángulos de orientación y de sus velocidades, realiza el cálculo de todos los algoritmos de estabilidad incorporados. Una vez realizados los cálculos los transmite por el bus CAN al microcontrolador que simula el vehículo.

En la Figura 4 se muestra un subconjunto de los datos que han sido transmitidos entre los dos dispositivos. En la primera de las columnas se muestra la velocidad recibida, en $\mathrm{m} / \mathrm{s}$, y en las siguientes los índices de estabilidad $\mathrm{SI}_{\mathrm{LONG}}, \mathrm{SI}_{\mathrm{LATS}}$, $\mathrm{SI}_{\text {LATT }}, \mathrm{SI}_{\mathrm{COM}}$ y $\mathrm{SI}_{\text {DIN }}$, que se transmiten. Como se observa, el índice de estabilidad $\mathrm{SI}_{\text {latt }}$ disminuye desde el valor 96.71 a 87.93 . Esta disminución se debe exclusivamente al aumento de la velocidad simulada del tractor, tal y como se puede comprobar en la ecuación (5). También se observa como $\mathrm{SI}_{\mathrm{COM}} \mathrm{y}$ SI $_{\text {DIN }}$ muestran valores similares y de menor magnitud. Esto es debido a que la prueba se realizó en una situación estática donde el prototipo estaba inclinado a lo largo del eje longitudinal (variación del ángulo de pitch). 


\subsection{FILTRADO DE DATOS}

Durante el diseño del prototipo se ha comprobado la necesidad de implementar un filtrado de datos. Al realizar la prueba anteriormente descrita, se pudieron observar variaciones bruscas en las velocidades angulares al realizar cambios bruscos en la orientación del equipo. En una prueba real esto también puede ocurrir debido a vibraciones del vehículo producidas por irregularidades del terreno. Para el filtrado de estas medidas, se implementó un filtro de Butterworth de segundo orden.

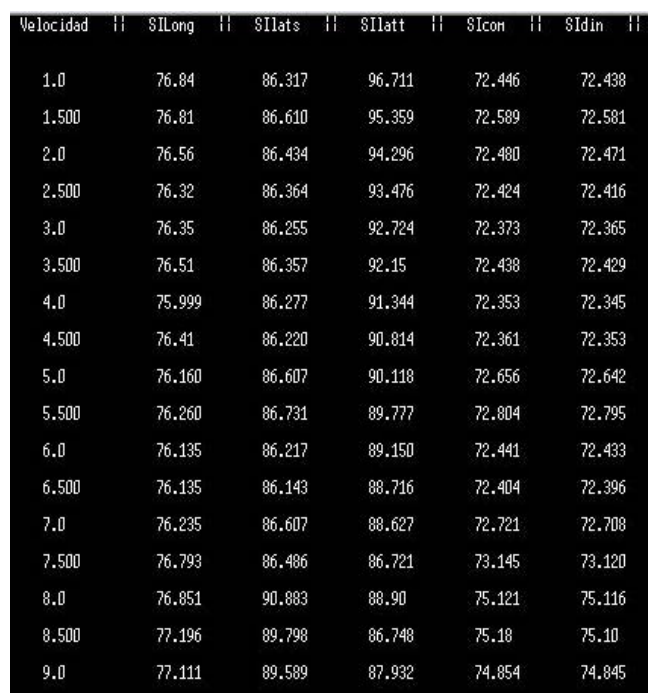

Figura 4: Transmisión a través de bus CAN

Para la realización de esta prueba se han almacenado datos filtrados $y$ sin filtrar, mientras que el dispositivo se somete a movimientos bruscos, pretendiendo de este modo simular vibraciones. El filtrado de las velocidades angulares es el más importante, ya que cuando el vehículo se somete a vibraciones es posible que se active una falsa alarma.

En la Figura 5 se puede observar la representación de dos señales de una de las velocidades angulares medidas durante una de las pruebas realizadas. Una de ellas es la señal de velocidad angular obtenida del sensor sin filtrar (en color rojo) y la otra señal (en color azul) es esta misma pero filtrada. En la señal sin filtrar se aprecian cambios bruscos y picos de gran magnitud que provocarían la activación de la alarma del prototipo, indicando la inestabilidad del vehículo de forma errónea. Por el contrario la señal filtrada muestra una respuesta más suave, atenuando considerablemente la magnitud de los cambios bruscos mencionados.

En la Figura 6 se comprueba el efecto del uso del filtro con el índice de estabilidad dinámico SI DIN. Se puede apreciar un número elevado de alarmas erróneas producidas sin utilizar el filtrado de datos (en rojo) y como estas falsas alarmas se eliminan mediante el filtrado de datos (en negro). Por tanto se verifica la necesidad de la implementación de filtros en las variables medidas.

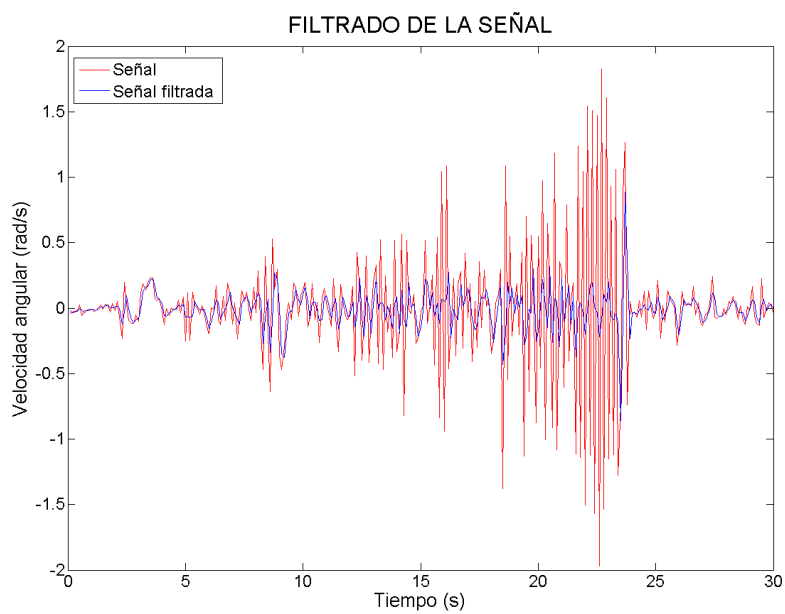

Figura 5: Velocidad angular filtrada

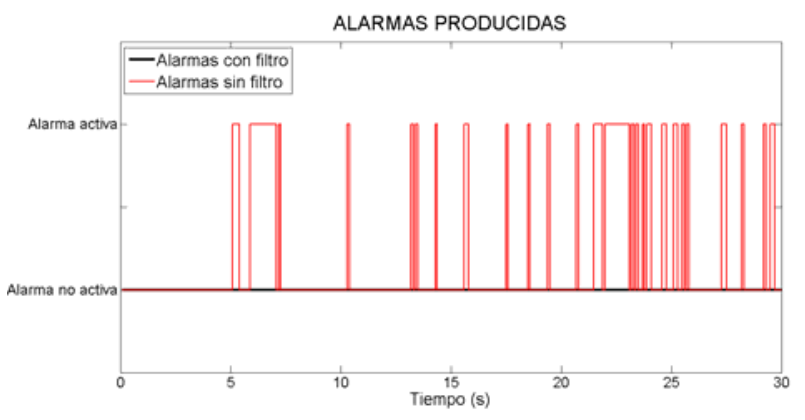

Figura 6: Alarmas producidas con filtro

\subsection{COMPARACIÓN DEL CÁLCULO DE ÍNDICES DE ESTABILIDAD}

Para asegurar que el dispositivo funciona correctamente, se ha realizado una comparación entre las alarmas generadas por el dispositivo ejecutando los algoritmos en tiempo real y las calculadas en Matlab a partir de los datos de ángulos y velocidades almacenadas en la tarjeta SD, considerando además los mismos parámetros mecánicos del vehículo.

El prototipo se sometió a diferentes movimientos simulando el movimiento que tendría si estuviera instalado en un vehículo industrial (inclinaciones laterales y longitudinales) llegando en varias ocasiones al límite a partir del cual el dispositivo detecta inestabilidad, provocando de esta manera la activación de la alarma objeto de esta comparación. En la Figura 7 se muestran las alarmas producidas en tiempo real en el dispositivo (en color rojo) y las alarmas calculadas posteriormente mediante Matlab. 
Como se puede observar, las alarmas obtenidas mediante ambas son coincidentes, por lo que el dispositivo realiza el cálculo de los algoritmos de estabilidad correctamente, siendo el tiempo de ejecución suficiente.

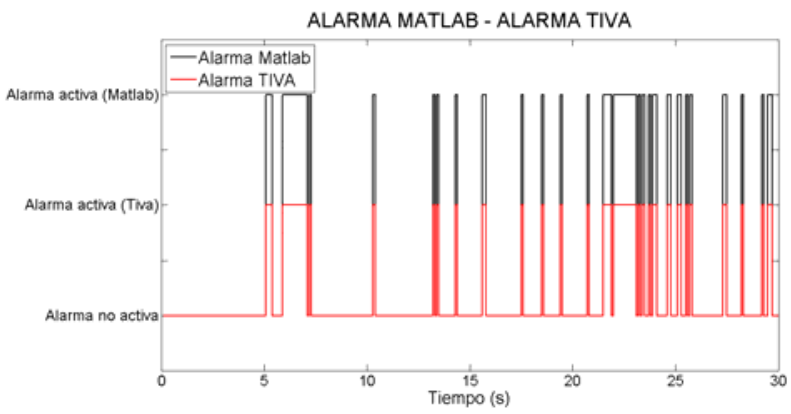

Figura 7: Comparación de alarmas en tiempo real

\subsection{PRUEBA CON VEHÍCULO AGRÍCOLA}

En este apartado se comenta la prueba realizada con el dispositivo en una situación real. Esta prueba se ha llevado a cabo mediante la utilización de un tractor, concretamente con el modelo New Holland 7.185, mostrado en la Figura 8.

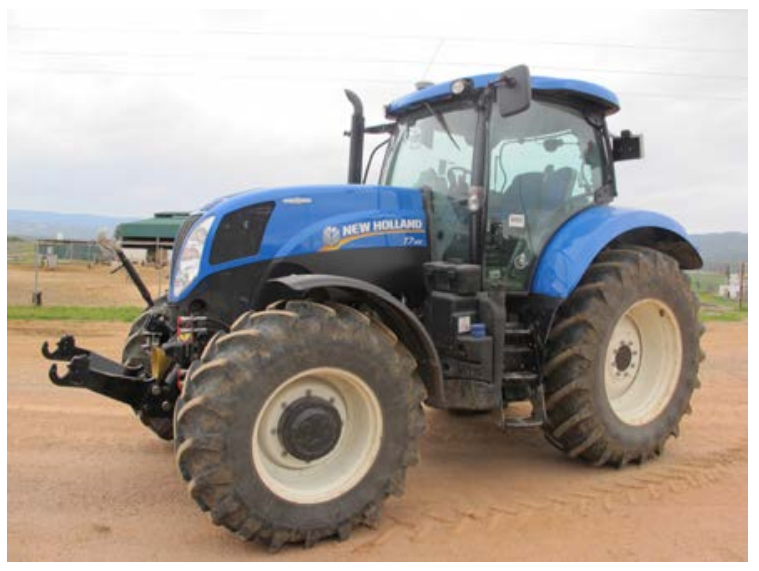

Figura 8: Tractor NEW HOLLAND T7.185

En la Figura 9 se muestra el lugar donde se colocó el dispositivo dentro del tractor, una zona plana cercana al operario y al centro de gravedad del tractor. La prueba consistió en realizar un recorrido con el vehículo sobre un terreno irregular con el fin de comprobar el funcionamiento del dispositivo $\mathrm{y}$ realizar un análisis posterior, utilizando para ello los datos registrados en la tarjeta $\mathrm{SD}$ durante el trayecto realizado.

En las Figuras 10 y 11 se muestra la evolución de los ángulos de orientación (roll y el pitch) y sus velocidades angulares. En estas gráficas se observa que los valores de los ángulos y de sus velocidades angulares no alcanzan valores elevados, sin sobrepasar los límites de los ángulos y velocidades críticos por lo que el dispositivo no detecta una situación de inestabilidad.

Por otra parte, en el tramo [420, 540] se aprecia que los ángulos de orientación son constantes y las velocidades angulares nulas. Este intervalo se corresponde a un periodo de tiempo en el que el tractor fue detenido durante la prueba. Los ángulos de orientación no nulos indican que el tractor se detuvo en un terreno en el que existía pendiente transversal y longitudinal.

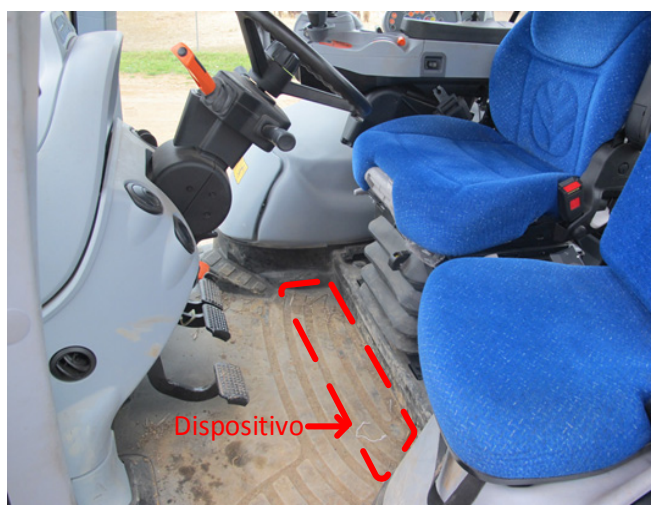

Figura 9: Colocación del dispositivo en el tractor

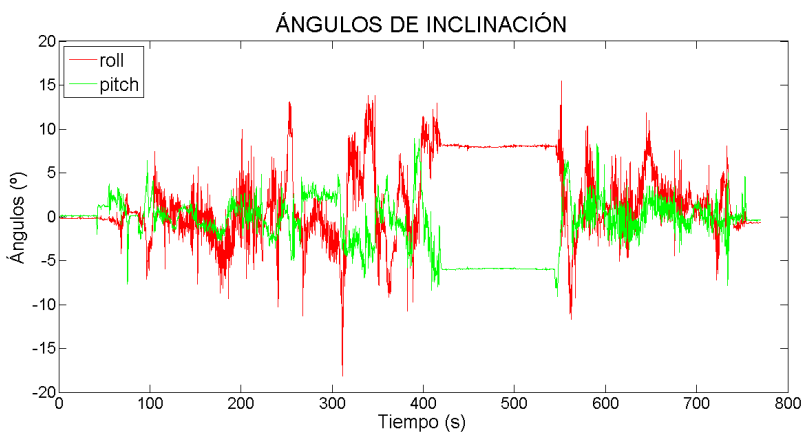

Figura 10: Ángulos de orientación

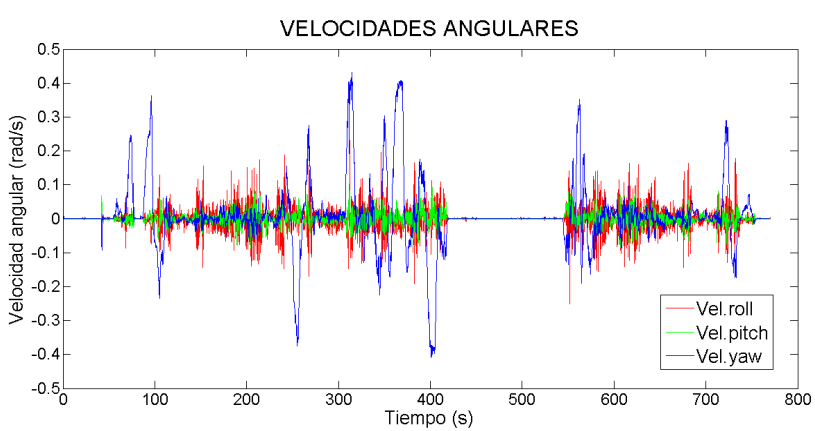

Figura 11: Velocidades angulares

En la Figura 12 se muestra la evolución de los índices de estabilidad. Se puede observar que ningún 
índice calculado indica la inestabilidad del tractor ni valores próximos a esta. En la Figura 12 también se puede observar el tramo en el cual el tractor se detuvo, donde los índices de estabilidad son constantes. El valor de estos índices en dicho tramo no indica la estabilidad máxima del vehículo (100) debido a que los ángulos de roll y pitch no son nulos y como se ha expuesto intervienen en el cálculo de todos los índices de estabilidad mostrados en este trabajo.

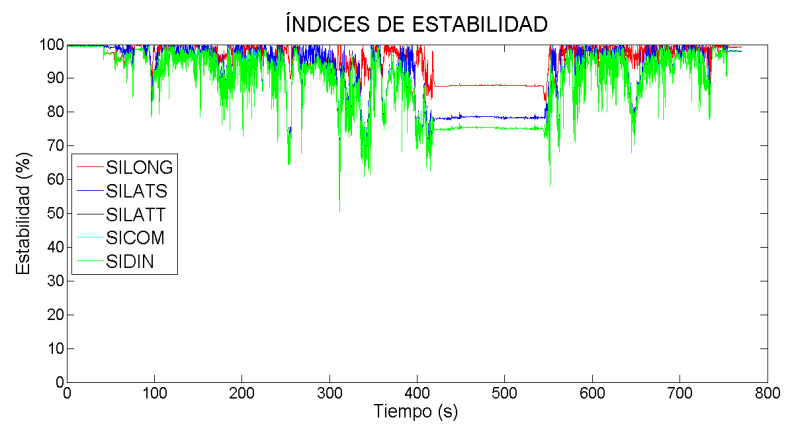

Figura 12: Índices de estabilidad

\section{CONCLUSIONES}

En este trabajo se ha desarrollado un prototipo de sistema anti-vuelco para vehículos industriales, y cuyo principal objetivo es el de servir como plataforma para el estudio y desarrollo de diferentes algoritmos de estabilidad. Se ha dotado al equipo de capacidad de registro, de una alarma sonora, una salida a relé y una interfaz gráfica que permite configurarlo y visualizar los índices de estabilidad.

El prototipo diseñado calcula en tiempo real un conjunto de índices de estabilidad y los transmite a través del protocolo CAN, lo que permite integrarlo con los sensores y mecanismos de control del propio vehículo donde se instale. Los resultados obtenidos en las pruebas realizadas, tanto en simulación como reales, ponen de manifiesto la importancia del desarrollo e investigación de este tipo de dispositivos en la reducción de accidentes por vuelco.

Como trabajo futuro se tiene previsto profundizar en el estudio de los algoritmos de estabilidad y su desempeño en diferentes vehículos. Uno de los aspectos más importantes de este tipo de sistemas es su robustez, ya que el disparo de alarmas o avisos no justificados hacen que el equipo pierda su validez como dispositivo de seguridad. Se hace necesario un estudio detallado de las diferentes vibraciones que se puedan dar en función de la tarea que desarrolle el vehículo y corroborar si el filtrado actualmente implementado es suficiente. Otros aspectos también de relevancia son la calibración del dispositivo y su emplazamiento en el vehículo donde se instale, de manera que siempre proporcione la orientación real. Además, si bien se ha podido comprobar que el tiempo de cálculo para los índices de estabilidad es despreciable respecto al periodo de muestreo empleado ( $0.1 \mathrm{~s})$, es necesario realizar un estudio exhaustivo del tiempo de respuesta del dispositivo ante situaciones reales de vuelco y verificar de esta manera la robustez del prototipo. También, se pretende dotar al sistema de una interfaz de comunicación adicional, como el GPRS (General Packet Radio Service).

\section{Referencias}

[1] Subgrupo de trabajo de Siniestralidad en la Agricultura, (2009) "Análisis de la siniestralidad en el sector agrario". Comisión Nacional de Seguridad y Salud en el Trabajo.

[2] Murphy, M. D., (2012), "Agricultural vehicle autopilot rollover risk assessment system", patente, $\mathrm{n}^{\mathrm{o}}$ : US 8,275,516 B2.

[3] Bulent, A., Liu, B., (2013) "Demonstrating tractor rollover stability using Lego mindstorms and smartphones", Journal of Agricultural Systems, Technology and Management, vol. 24, pp.1-11.

[4] Liu, B., Bulent, A., (2013) "SafeDriving: A mobile application for tractor rollover detection and emergency reporting", Computers and Electronics in Agriculture, vol. 98, pp. 117-120.

[5] Liu, J., Ayers, P.D., (1998) "Application of a tractor stability index for protective structure deployment", Journal of Agricultural Safety and Health, Special issue (1), pp. 171-181. 